antidotes and treatments that should be performed, as well as risk factors and strategies to prevent extravasation.

Materials and Methods A literature review was performed, through research and analysis of guidelines and articles obtained from PubMed since January/2000 to September/2012, intersecting the terms 'cytotoxic extravasation', 'chemotherapy extravasation' and 'extravasation treatment'. The summary of product characteristics of all of intravenous cytotoxics available in Portugal was also reviewed. Some holders of market authorization were also contacted whenever we considered additional information was required.

Results A total of 42 intravenous antineoplastics available in Portugal were analysed, distributed as follows based on tissue injury after extravasation: 16 vesicant products, 16 irritants and 10 neutral products. A summary table was created with the risk factors (e.g., vesicant drugs, higher drug concentrations, previous vinca alkaloids, elderly, impaired sensory perception, generalised vascular disease) and measures that prevent extravasation (e.g. ensure that the IV site can be clearly visualised, do not use a butterfly needle with a vesicant drug). Nine individual algorithms were developed, according to the latest guidelines, which guide the work of healthcare professionals in case of extravasation (e.g., measures for immediate treatment, applying heat/cold, recommended antidote and instructions for its use). A list was drawn up with all cytotoxics, each being identified with a colour, which corresponded to the colour of the separator with the algorithm to treat its extravasation. An extravasation kit was also designed and a model for document the appropriate recording of extravasation and clinical monitoring of the patient. Conclusions The guidelines developed are a valuable tool for all hospital services that prepare and administer injectable chemotherapy, contributing to responding quickly and effectively to episodes of extravasation.

No conflict of interest.

\section{GRP-080 HAEMATOLOGICAL TOXICITY SECONDARY TO TREATMENT WITH DIAZOXIDE: A CASE REPORT}

doi:10.1136/ejhpharm-2013-000276.080

MD Alonso Castañé, A Martin Sanz, C Guindel Jimenez, J Ortiz de Urbina, I Yáñez González, M Sáez Villafañe. Complejo Asistencial Universitario de León, Farmacia, León, Spain

Background Hyperinsulinism is a rare cause of persistent hypoglycaemia in the neonatal period. Tolerance of diazoxide is usually excellent.

Purpose To describe a case of normocytic anaemia secondary to treatment with diazoxide in an infant diagnosed with congenital hyperinsulinism.

Materials and Methods A retrospective review of medical records from admission in February 2012 to the current situation and a PubMed search of possible cases of this adverse effect.

The patient was a 17-day-old infant who was treated with diazoxide (maximum dose $25 \mathrm{mg} / \mathrm{kg} /$ day) with good response, allowing the progressive discontinuation of the IV glucose and glycaemia control. The patient was started at a dose of $45 \mathrm{mg} /$ day which has been increased to the current dose of $140 \mathrm{mg} /$ day to encourage weight gain.

Results Normocytic and normochromic anaemia gradually because established with tachycardia (decrease in $\mathrm{Hb}$ from $14 \mathrm{mg} / \mathrm{dl}$ to $8.7 \mathrm{mg} / \mathrm{dL}$ in 15 days), which was thought to be associated with diazoxide, as the other parameters were within normal ranges (echocardiography, thyroid function, iron deficiency study).

The haematological toxicity of diazoxide to be usually thought to be neutropenia and thrombocytopenia although anaemia is rarely described. This reaction was notified by the yellow card scheme to the regional pharmacovigilance system. An evolutionary anaemia study was carried out and monitored by transfusions of packed erythrocytes.

Results Hematologic toxicity is thought to be dose dependent and indicates withdrawal of the drug. After a benefit/risk review, the patient is currently still on diazoxide, although other possible treatment options were raised.

After a search in PubMed, we found a single case of anaemia and febrile neutropenia secondary to treatment with diazoxide in an adolescent with hyperinsulinism which was resolved after withdrawal of the drug. This adverse effect may be considered odd.

The importance of Pharmacy Services and other health professionals in reporting adverse reactions is appreciated for the safe use of drugs.

No conflict of interest.

\section{GRP-081 HEALTH INFORMATION TECHNOLOGY AND STRESSORS HOW TO MEASURE AND ELIMINATE THEM}

doi:10.1136/ejhpharm-2013-000276.081

${ }^{1} \mathrm{M}$ Fleury, ${ }^{2} \mathrm{~L}$ Bouchoud, ${ }^{1 P}$ Bonnabry. 'Geneva University Hospitals, Pharmacy/School of pharmaceutical sciences/University of Geneva/University of Lausanne, Geneva, Switzerland; ${ }^{2}$ Geneva University Hospitals, Pharmacy, Geneva, Switzerland

Background The daily tasks of hospital personnel are regularly interrupted. Unexpected breaks in work patterns act as supplementary cognitive burdens on health workers (hence the term stressors) and can lead to errors because they break up the logical flow of clinical tasks.

Purpose To examine whether the introduction of Health Information Technology (HIT) (CytoAdmin - a scanning system for matching patients to their chemotherapy treatment protocols) to a cancer outpatient unit had any immediate effect on stressors, with the broader aim of then reducing their types and frequency.

Materials and Methods Based on techniques drawn from the field of Human Factors and Ergonomics (HF\&E) [1], we established a protocol for carrying out ergonomic evaluation and measuring stressors. The System Engineering Initiative for Patient Safety model was our guiding principle [2]. The protocol covered all tasks in the unit and included field observations, listing stressors (number, type) observed during the introduction of the HIT, and suggesting process redesign methodologies.

Results During the first 6 days of CytoAdmin's introduction, we carried out $31 \frac{1}{2}$ hours of observation of stressors and identified 89 different types (2.7 stressors/hour). The HIT itself generated 21 new stressors (24\% of the total). Amongst these were the insufficient number of computers needed to complete tasks, technical hardware problems and the inclusion of scanning in a wellestablished daily care routine. Ergonomic redesign of workflows allowed us to neutralise all new stressors. Other major stressors were telephone calls (13 types of stressors, 15\%), followed by consulting a physician ( 9 types, $10 \%$ ).

Conclusions The introduction of this HIT increased the number of stressors by creating new ones. The HF\&E system developed was efficient at detecting new stressors, redesigning the process and eliminating them. Although these methodologies are timeconsuming, ergonomic evaluations are essential for the satisfactory and safe use of newly-introduced HIT.

\section{References}

1. Carayon P. (Editor) Handbook of Human Factors and Ergonomics in Health Care and Patient Safety, $2^{\text {nd }}$ edition, Lawrence Erlbaum Associates, Hillsdale, NJ, 2012.

2. Carayon P, et al, Work system design for patient safety: The SEIPS model. Qual Saf Health Care 2006;15(1):50-58

No conflict of interest. 